Kompass

Pneumologie

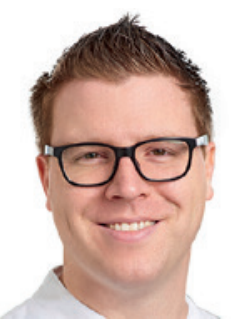

\section{Simon-Dominik Herkenrath}

Krankenhaus Bethanien gGmbH, Klinik für Pneumologie und Allergologie, Solingen, Deutschland

\title{
Interaktionen der Lunge: Funktionelle Einheiten erfassen und beachten
}

Kardiale und pulmonale Erkrankungen haben eine hohe Prävalenz und beeinflussen die Morbidität sowie Mortalität eines Menschen entscheidend. Kommt es in diesem Zusammenhang zu einer koexistenten Funktionseinschränkung beider Organsysteme, führt dies jedoch nicht nur zu einer aggravierten Beschwerdesymptomatik, sondern ist auch prognostisch besonders ungünstig. Eindrucksvoll zeigt sich dies zum Beispiel bei Koexistenz einer Herzinsuffizienz und einer chronisch-obstruktiven Lungenerkrankung (chronic obstructive pulmonary disease, COPD) [1-3]. Das Zusammenspiel von Herz und Lunge ist Voraussetzung für einen suffizienten Gasaustausch und eine stabile Hämodynamik. Wenn eines der beiden Organe funktionelle Einschränkungen aufweist, gibt es zwar wechselseitige Kompensationsmechanismen, im Langzeitverlauf führen pulmonale Erkrankungen jedoch häufig auch zu einem kardialen Funktionsverlust, und umgekehrt $[4,5]$.

Die hohe Prävalenz von Endorganschäden im Bereich der Lunge und des Herzens beruhen neben überlappenden Risikofaktoren (Alter, Tabakkonsum usw.) auch zum Teil auf gemeinsamen Pathomechanismen, bei denen insbesondere dem chronisch-inflammatorischen Milieu eine besondere
Rolle zukommt [6-8]. So wirken inflammatorische Zytokine bei der COPD zum einen direkt im Bereich des Lungenparenchyms, aber ebenso an den glatten Muskelzellen und Endothelzellen der Arterien. Dadurch kommt es nicht nur im Bereich der Pulmonalarterien zu einer endothelialen Dysfunktion und einem Gefäßumbau mit Erhöhung des pulmonalvaskulären Widerstands, sondern auch systemisch, mit Ausbildung einer arteriellen Hypertonie, linksventrikulären Hypertrophie, diastolischen Dysfunktion, einem Anstieg des linksventrikulären enddiastolischen Drucks und einer pulmonalvenösen Stauung. Die Schädigung des Lungenparenchyms führt indes zu einer progressiven Atemflusslimitation mit «air trapping», Überblähung sowie Hypoxievermittelter Vasokonstriktion. Der ansteigende Druck im Thorax vermindert den venösen Blutfluss zum Herzen und führt so zu einer Verminderung des Schlagvolumens und des Herzindex. Diese komplexen wechselseitigen Effekte zwischen Lunge und Herz erklären die hohe Prävalenz koexistenter Endorganschäden und fordern einen ganzheitlichen therapeutischen Ansatz.

Das Kardinalsymptom einer kardialen oder pulmonalen Erkrankung ist häufig die Belastungsdyspnoe. Zwar ist die kardiologische und pneumologische Basisdiagnostik in vielen Fällen wegweisend, kommt es jedoch zur koexistenten Erkrankung in beiden Organsystemen oder zu überlappenden Endorganmanifestationen im Rahmen einer Systemerkrankung, hilft oft erst die Spiroergometrie in der Einordnung pathologischer Einflussgrößen und Akzente. Welche prognostische Bedeutung spiroergometrische Parameter bei der systemischen Sklerose haben können, erläutert Stefan Krüger anhand der Arbeit von Ralf Ewert und Kollegen. Neben einer reduzierten Peak-Sauerstoffaufnahme erweist sich auch ein $\mathrm{VE} / \mathrm{VCO}_{2}$-slope $>35$ als prognostisch ungünstig.

Die Steigung der Kurve von Atemminutenvolumen im Verhältnis zur $\mathrm{CO}_{2}$-Elimination begleitet uns auch bei der pulmonalen $\mathrm{Hy}$ pertonie seit einiger Zeit, da sie sich als Indikator für eine pulmonalvaskuläre Erkrankung herausgestellt hat. Sowohl die pulmonal-arterielle Hypertonie (PAH) als auch die chronisch-thromboembolische pulmonale Hypertonie (CTEPH) werden medikamentös aktuell ausschließlich in Form einer Vasodilatation behandelt. Neuere zahlreiche vielversprechende präklinische Studien verfolgen jedoch seit einiger Zeit immunmodulatorische Ansätze. Diese werden das therapeutische Spektrum in der Behandlung der PAH und CTEPH in Zu- karger@karger.com www.karger.com/kkp

(c) 2020 S. Karger GmbH, Freiburg

Karger"
Simon-Dominik Herkenrath

Krankenhaus Bethanien gGmbH

Klinik für Pneumologie und Allergologie

Aufderhöher Straße 169-175, 42699 Solingen, Deutschland

simon-dominik.herkenrath@klinik-bethanien.de 
kunft möglicherweise grundlegend ändern. Einblick in diese spannende Thematik geben uns Thomas Koudstaal und Mitarbeitende in ihrer Übersichtsarbeit zur immunologischen Perspektive bei PAH und CTEPH.

Infolge der Interaktion von Herz und Lunge können Therapien in vielerlei Hinsicht positiv wirken. Dies zeigt zum Beispiel die medikamentöse Deflation einer überblähten Lunge mittels LABA und LAMA. Hierdurch

\section{Literatur}

1 Canepa M, Temporelli PL, Rossi A, et al.: Prevalence and Prognostic Impact of Chronic Obstructive Pulmonary Disease in Patients with Chronic Heart Failure: Data from the GISSI-HF Trial. Cardiology. 2017;136(2): 128-137.

2 Hawkins NM, Virani S, Ceconi C: Heart failure and chronic obstructive pulmonary disease: the challenges facing physicians and health services. Eur Heart J. 2013;34(36): 2795-2803.

3 Canepa M, Straburzynska-Migaj E, Drozdz J, et al.: Characteristics, treatments and 1-year prognosis of hospitalized and ambulatory heart failure patients with chronic obstructive pulmonary disease in the European Society of Cardiology Heart Failure Long-Term Registry. Eur J Heart Fail. 2018;20(1):100-110. kommt es bei Patienten mit einer COPD nicht nur zu einer Besserung der Hyperinflation und Atemmechanik, sondern sekundär auch zu einer Zunahme des pulmonalen Blutflusses, des linksventrikulären enddiastolischen Volumens sowie des Schlagvolumens $[9,10]$.

Interaktionen zwischen Herz und Lunge sind komplex, erfordern eine differenzierte Auseinandersetzung mit pathophysiologischen Akzenten, bieten aber auch die
Chance einer therapeutischen Intervention, die nicht nur einem Organ zugutekommt.

Eine anregende Lektüre zum Thema kardiopulmonale Interaktionen wünscht Ihnen

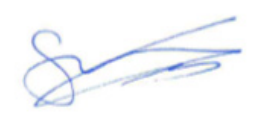

Simon-Dominik Herkenrath
4 Canepa M, Franssen FME, Olschewski H, et al.: Diagnostic and Therapeutic Gaps in Patients With Heart Failure and Chronic Obstructive Pulmonary Disease. JACC Heart Fail. 2019;7(10):823-833.

5 Magnussen H, Canepa M, Zambito PE, et al.: What can we learn from pulmonary function testing in heart failure? Eur J Heart Fail. 2017; 19(10):1222-1229.

6 Kovacs G, Agusti A, Barberà JA, et al.: Pulmonary Vascular Involvement in Chronic Obstructive Pulmonary Disease. Is There a Pulmonary Vascular Phenotype? Am J Respir Crit Care Med. 2018;198(8):1000-1011.

7 Barnes PJ: Inflammatory mechanisms in patients with chronic obstructive pulmonary disease. J Allergy Clin Immunol. 2016;138(1): 16-27.
8 Koudstaal T, Boomars KA, Kool M: Pulmonary Arterial Hypertension and Chronic Thromboembolic Pulmonary Hypertension: An Immunological Perspective. J Clin Med. 2020;9(2).

9 Hohlfeld JM, Vogel-Claussen J, Biller H, et al.: Effect of lung deflation with indacaterol plus glycopyrronium on ventricular filling in patients with hyperinflation and COPD (CLAIM): a double-blind, randomised, crossover, placebo-controlled, single-centre trial. Lancet Respir Med. 2018;6(5):368-378.

10 Vogel-Claussen J, Schönfeld C-O, Kaireit TF, et al.: Effect of Indacaterol/Glycopyrronium on Pulmonary Perfusion and Ventilation in Hyperinflated Patients with Chronic Obstructive Pulmonary Disease (CLAIM). A Double-Blind, Randomized, Crossover Trial. Am J Respir Crit Care Med. 2019;199(9): 1086-1096. 